\title{
Didactical situations to treat Lorentz and Galileo transformations in theoretical physics
}

\author{
S. A. de Souza Farias \\ Institute of Educational Sciences, Federal University of Western Pará, Santarém, Brazil. \\ e-mail: sergio.farias@ufopa.edu.br \\ D. P. Meira Filho \\ Federal Institute of Pará, Campus Santarém, Santarém, Brazil. \\ e-mail:damiao.meira@ifpa.edu.br \\ J. K. Santos Kamassury \\ Federal University of Santa Catarina, Florianópolis, Brazil.
}

Received 19 July 2021; accepted 19 August 2021

\begin{abstract}
Notions of electromagnetism and special theory of relativity, require important mathematical knowledge applied to theoretical physics. Recognizing pedagogical difficulties in the teaching of theoretical physics, the theory of didactical situations, which consists of a set of practices that aim to contribute to the improvement of mathematics teaching. In this context, the present work is motivated to present a set of practices based on the theory of didactical situations with a focus on teaching Electromagnetism and Special Theory of Relativity, where problems that require an understanding of the Galileo and Lorentz transformations. Specifically, the didactic situation is constructed by means of four problem proposals, while in the adidatic situation, the student is invited to understand the roles of these transformations in the study of these problems. Ultimately, the relevance of the educator in the institutionalization situation is reinforced, a moment when it must be clarified how all mathematical relations are strongly related to physical principles.
\end{abstract}

Keywords: Electrodynamics; Special Theory of Relativity; Galileo and Lorentz transformations; didactic situations.

DOI: https://doi.org/10.31349/RevMexFisE.19.010204

\section{Introduction}

The 19th century is characterized by immense theoretical advances in the field of physics [1]. In this context, James Clerk Maxwell (1831-1879), physicist and mathematician, managed to synthesize the laws of electromagnetism in a concise mathematical formulation better known as Maxwell's Equations [2]. Another important name for the consolidation of electromagnetism was Hendrik Antoon Lorentz (1853-1928), who formulated the equation that explains the relationship between electrical and magnetic force [3]. As in the 19th century, at the beginning of the 20th century, another important theory is presented to the scientific community at the time [4], this is due to the German theoretical physicist of Jewish origin, Albert Einstein (1879-1955).

Maxwell's equations express the laws of electrodynamics; the first and second equations express Gauss's laws for the electric and magnetic field successively. The third equation is the result of experiments carried out by Michael Faraday (1791-1867) and Joseph Henry (1797- 1878) and the fourth equation is Ampère's law plus a term for the displacement current.

Maxwell admitted that the electric and magnetic fields oscillate perpendicularly and in phase, so is the characterization of electromagnetic radiation. With this admission, Maxwell showed that the speed of propagation of electromagnetic radiation in a vacuum is equal to the speed of light propagation.
Later (1887) the "Maxwell waves" were verified experimentally by Heinrich Rudolph Hertz (1857-1894) [5].

When the value of a quantity has the same value in different inertial reference frames, considering a certain set of transformations, that quantity is said to be invariant. In the context of electromagnetism, the propagation speed of electromagnetic radiation is invariant under Lorentz transformation between two different inertial frames.

It is worth noting that the algebraic structure of physical laws ought to be preserved for all different inertial reference frames. For example, the algebraic structure of Maxwell's equations is unchanged when they are subject to Lorentz transformations, therefore is adequate to say that Maxwell's equations are covariant.

The laws of physics are expressed in sets of transformations between inertial references. Galileo's transformations (GTs) support the covariance of the laws of dynamics, but they do not support the covariance of the laws of electromagnetism.

All of these questions require a great deal of knowledge of physics and mathematics, but they are, nonetheless, covered since high school. The present work aims to: discuss: i) the limitations of Electrodynamics and the role of Lorentz transformations for the solution of these limitations; ii) how the Special Theory of Relativity (STR) arises from the limitations of electrodynamics and iii) a didactic sequence capable 
of addressing the relationship between electrodynamics and STR.

The didactic sequence intends to expose students to the conceptual changes that arise when GTs are applied between two observers in different inertial frames of reference. This phenomenological approach consists of four situations that will show the student the limitations of electrodynamics and how a new set of transformations demand a new theory of physics, the special theory of relativity.

In the theory of didactic situations (TDS), formulated by Guy Brousseau [6], it is necessary to identify a medium (milieu) in which the didactic situation is present. According to Paulo Jorge Magalh aes Teixeira and Claudio Cesar Manso Passos [7]:

This theory has, as one of the primary objectives of didactics of mathematics, the characterization of a learning process by means of a series of reproducible situations, called didactic situations, which establish the determining factors for the evolution of students' behavior.

We observed that four fictitious situations can make the student to question the validity of the Galileo's transformations, which make manifest the covariance of the laws of mechanics. The challenge is to ensure that the physical laws be covariant in the face of a new set of transformations, namely, the called Lorentz transformations. The proposed TDS ( $m i$ lieu) has its core in this challenge.

The first fictitious situation was extracted from a high school teaching book, the second and third situations were extracted from higher education books, and the fourth situation was proposed by the authors of the present text.

The model of interaction between the subject and the milieu is constructed using an explanatory text on theoretical physics that requires an advanced knowledge of mathematics. To this end, the student must overcome limits and thus acquire new skills.

The TDS set of practices aims to lead the student to seek autonomy, and this is done in didactic, adidactic, and institutional situations. The didactic situation occurs in the process of understanding of the validity of the set of transformations facing the proposed situations. The adidactic situation occurs through the interaction of the subject with the milieu, the institutional situation occurs in the strong participation of the teacher, demonstrating the relationship between all the rules of applied Mathematics and the Physical principles that support CE and STR.

This text will discuss the covariance of electromagnetism equations, which Einstein called asymmetries [8] by means of four situations: i) the magic carpet; ii) loop on rails; iii) ether and iv) high-speed mirror. We intend to clarify how the STR connects with CE by means of the four situations.

In the didactic situation, the teacher presents the milieu and the rules of interaction between the subject and the milieu. This step consists of explaining four situations and the importance of choosing a set of transformations in inertial reference frames. The teacher must clarify that the laws of physics should not depend on whether an inertial frame is at rest or in a rectilinear and uniform motion.

The suggested dynamics can be applied in higher education courses for teachers of Mathematics and Physics.

\section{Problems and didactic sequence}

\subsection{Step 1}

In step 1, the teacher is the protagonist and explains four hypotheses and the possible results of the application of GTs in these situations. The teacher needs to clarify that GTs are the appropriate transformations used in the context of classical dynamics of Newton, and that when applied to electrodynamics, they make Maxwell's equations, electromagnetic wave equations, and Lorentz's strength, non-covariant equations.

In the TDS, GTs are part of the students' prior knowledge. The teacher needs to point out that the laws of electrodynamics need to be supported by transformations to inertial reference frames. Step 1 consists of qualitatively manipulating the laws of electrodynamics with the same transformations already known by the students.

In situation 1 (magic carpet, see Fig. 1) when we use the GT we have that the girl understands that the electric charge moves with speed, but the boy does not perceive speed for the electric charge. Hence, Lorentz's strength would depend on the frame of reference.

In situation 2, both observers in Fig. 2, located in different inertial frames, perceive the electromotive force emf but differ about the cause of the emf. Thus, there is an apparent contradiction in what each observer agrees is the source of the emf if GTs are applied for the spiral on rails. It should be noted that the emf is described using Maxwell's equations.

Situations 3 and 4 deal with the speed of light. The former describes the hypothesis of a means for the propagation of electromagnetic radiation (ether) that moves relative to inertial reference frames. The direct application of the GT predicts different radiation speeds depending on the movement of the inertial frame.

Situation 4 consists of a thought experiment that provokes the student to question the variance or invariance of the speed of the electromagnetic radiation (light). It is the most playful situation of the proposals because it raises the possibility of the boy not seeing his face reflected in the mirror.

These four situations raise some possible inconsistencies: i) can different observers observe different movements for the electric charge? ii) can the same emf be caused by different fields? iii) can the same value for the speed of light, in different inertial frames, make the ether hypothesis unsupported? v) how can I look in the mirror and not see my face?

These four situations help to understand the importance of the covariance of the electrodynamic equations. 


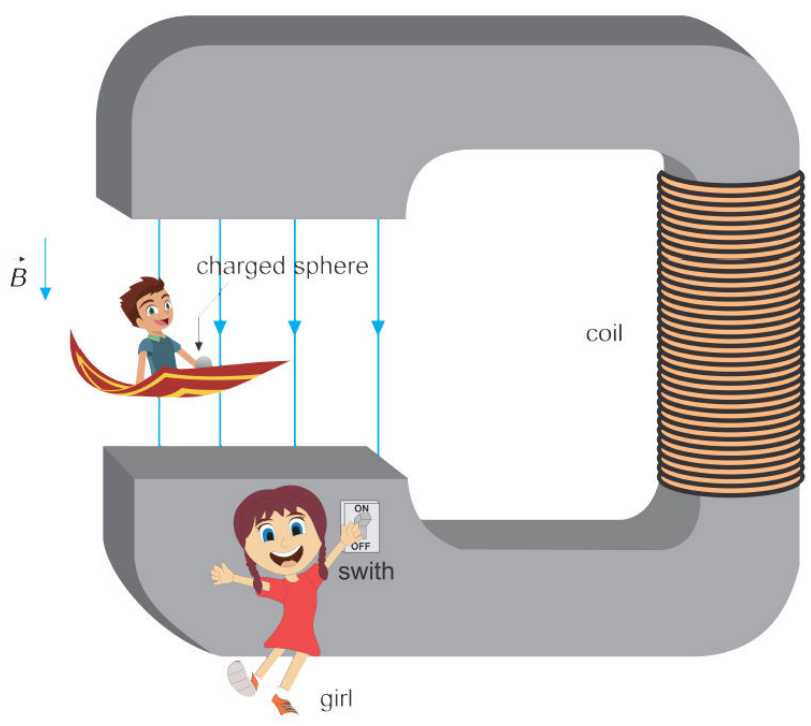

FIGURE 1. Transporting an electrically charged sphere, the boy flying on a magic carpet crosses a region where a constant and uniform magnetic field generated by the electromagnet acts.

\subsubsection{Magic carpet}

The magic carpet or ying carpet is well known from the tales that make up the Persian work One Thousand and One Nights.. However, here it will help us to understand the inconsistency in the description of Lorentz's force through Galileo's transformations. The following text was taken from a textbook that is used in the context of public high school [9]:

A boy flies on a magic carpet, close to the ground, with constant speed $\vec{v}$, horizontal, transporting a sphere with a positive electrical charge, supported without friction on an insulating pillow. At a given moment, it crosses through the poles of a large electromagnet that can generate a uniform and constant magnetic field, whose magnetic field vector $\vec{B}$ is oriented downwards. At that moment, beside the electromagnet, a girl standing on the ground turns on the electromagnet.

Figure 1 shows the situation in which the magnet field and the girl are fixed in frame $O$, the sphere and the boy are in frame $O^{\prime}$.

Analyzing the situation first concerning the girl, according to Lorentz's strength $\vec{F}=q \vec{v} \times \vec{B}$, there is the action of a magnetic force that points to the center of the circumference, according to the vector product $\vec{v} \times \vec{B}$.

In the boy's rest frame, the sphere has velocity $\vec{v}=\overrightarrow{0}$, remaining at rest on the cushion while the force $\vec{F}=q \vec{v} \times \vec{B}$, it is therefore null $\vec{F}=\overrightarrow{0}$ and $|\vec{F}|=0$.

\subsubsection{Spiral on rails}

Consider a conducting loop in a train car held by two people as shown in Fig. 2. When the train car moves between the poles of a giant magnet, it generates an electromotive force

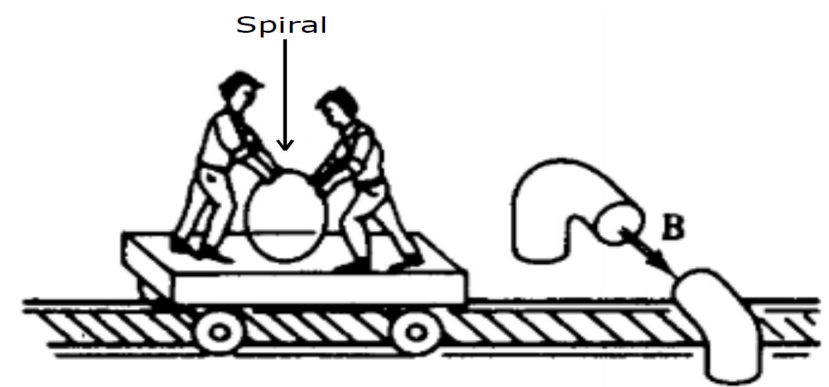

FIGURE 2. Spiral on rails [10].

(emf) that generates a current. As is known, the emf that arises is due to the action of the force $\vec{F}=q \vec{v} \times \vec{B}$, on the electrical charges that make up the structure of conducting loop.

According to David J. Griffiths [10]:

This emf is due to the magnetic force acting on the electrical charge in the wire loop that is moving along with the train. On the other hand, if someone naively applied the laws of electrodynamics to that system, what would be the prediction? No magnetic force because the coil is at rest. But as the magnet passes, the wagon's magnetic field will change and a varying magnetic field induces an electric field, according to Faraday's law. The resulting electric force would generate a emf in the loop given by: $\epsilon=-d \phi / d t$. Since Faraday's law and the flow rule provide for the same emf, people on the train will get the same answer, although their physical interpretation of the process is completely wrong.

The observer outside the train (frame $O$ ) observes a nonzero emf due to the magnetic field, while the observer on the train (frame $O^{\prime}$ ) observes an emf, due to the magnetic force equal to zero, but a resulting emf different from zero due to the electric field. Each frame of reference leads to different interpretations for the emf!

Until the removal of the different interpretations referring to the fem in the early 20th century, it was believed that one observer was right and the other wrong, and to determine the correct observer it would be necessary to admit that the electromagnetic fields consisted of deformations of an invisible medium (ether)[10]. Therefore, the laws of electrodynamics should be measured about this medium.

\subsubsection{Ether}

The consequent determination of the speed of light using permeability and permissiveness in a vacuum leads to the following question: about which frame of reference was this speed defined? Maxwell realized the need to incorporate the idea of the ether, bringing something hitherto dormant [11]. Regarding these issues Maxwell commented [12]: 
But in all these theories, the question naturally arises: If something is transmitted at a distance from one particle to another, what is its condition after it has left one particle and before reaching the other? If this something is the potential energy of the two particles, as in Neumann's Theory, how can we conceive of this energy as existing at a point in space, without coinciding with either one or the other particle? In reality, whenever energy is transmitted in time from one body to another, there must be a medium or substance in which the energy exists after it leaves one body and before it reaches the other, because energy, as Torricelli said, "is the quintessence of a nature so subtle that it cannot be contained in any vessel, except in the most intimate substance of material things". Therefore, all these theories lead to the conception of a medium through which propagation takes place, and if we accept this medium as a hypothesis, I think it should occupy a prominent place in our investigations, and we should strive to build a mental representation. of all the details of his action, as was my constant objective throughout this treaty.

In this way, the entire electromagnetic formulation rested under a medium called ether, and this had specific properties to support the vibrations of the waves such as mechanical properties that allowed the transmission of forces to matter through tensions, pressures, and rotations [13]. And this was the medium that was taken as a reference and, therefore, the reference whose speed of light was linked.

Admitting ether as a medium whose electromagnetic wave propagation was linked, also ended up considering the existence of a privileged reference in physics, in which the medium (ether) was at rest [11]. This privileged referential of the ether was against Galileo's Relativity in which the equivalence between the inertial references predominated so that it would not be possible to have an inertial frame better than the other inertial frame. But, according to what has been presented so far, about the existence or not of this luminous medium, it would be possible from optical experiments to infer as to its supposed existence.

The ether hypothesis would be confirmed by identifying the speed of the Earth's movement through the ether, with the aid of light propagation experiments these studies would be possible [14]. Such studies were well structured and some of the scientists of the time claimed to find positive results while others did not identify any effect regarding speed [15]. Here we will present important studies that prove the absence of ether.

An important study on the possible existence of the ether was carried out by Fresnel in 1817 and verified by Fizeau in 1851. According to Fresnel's hypothesis, if light propagates in a transparent medium at rest, then the speed of light measured by an observer at rest about ether is simply: $u=c / n$. If the ether is completely dragged by the transparent object then the measured speed of light about the ether is: $u=(c / n)+v$, where $v$ is the speed of the fully dragged transparent object and $n$ is the index of refraction. But if the ether is partially dragged by the transparent object, then a correction factor $\beta$ is associated with $u=(c / n)+\beta v$. Fresnel admitted that the correction factor $\beta$ depends on the density of the ether when entering the medium $\left(\rho_{e}\right)$ and on the density of the ether after penetrating the medium $\left(\rho_{f}\right)$ :

$$
\beta=\left(1-\frac{\rho_{e}}{\rho_{f}}\right) .
$$

Note that if $\rho_{e}$ is much smaller than $\rho_{f}$ then $\beta$ tends to zero, the ether is completely dragged. Thus the speed of light measured by a standing observer in relation to the ether, according to Fresnel's theory is:

$$
u=\frac{c}{n}+v\left(1-\frac{\rho_{e}}{\rho_{f}}\right) .
$$

This privileged reference frame of the ether was in direct conflict with the principles of Galileo's Relativity in which the equivalence between the inertial reference frames predominated so that it would not be possible to have an inertial frame better than the other inertial frame. The result (1) raises the question about a set of transformations that sustain (reinforce) it. The ether hypothesis would be confirmed by identifying the Earth's movement speed through the ether.

\subsubsection{High speed mirror}

In classical mechanics, the resulting velocity of two bodies consists of the sum of velocity, if the bodies are in the same direction with opposite senses. This the prevising of Galileo's transformations.

Let's imagine a boy moving at the speed of light in the same direction as the beam of light (see the Fig. 3). For this boy to see himself in the mirror, the beam must be reflected by the mirror. In this situation is it possible that the boy does not see himself in the mirror?

The answer to that question would be given in 1887 by Albert Michelson (1852-1931) and Edward Morley (18381923). They set up an experiment that aimed to detect the relative movement of the Earth through the ether.

If the speed of light does not depend on the inertial frame, we would be able to see the boy's face in the mirror even at high speeds. This thought experiment may lead students to think that the speed of light can be greater than $300,000 \mathrm{~km} / \mathrm{s}$.

The explanation for the result of the high-speed mirror mental experiment would come with the STR. The notions of space and time for bodies at speeds compared to the speed of light would be reformulated. 


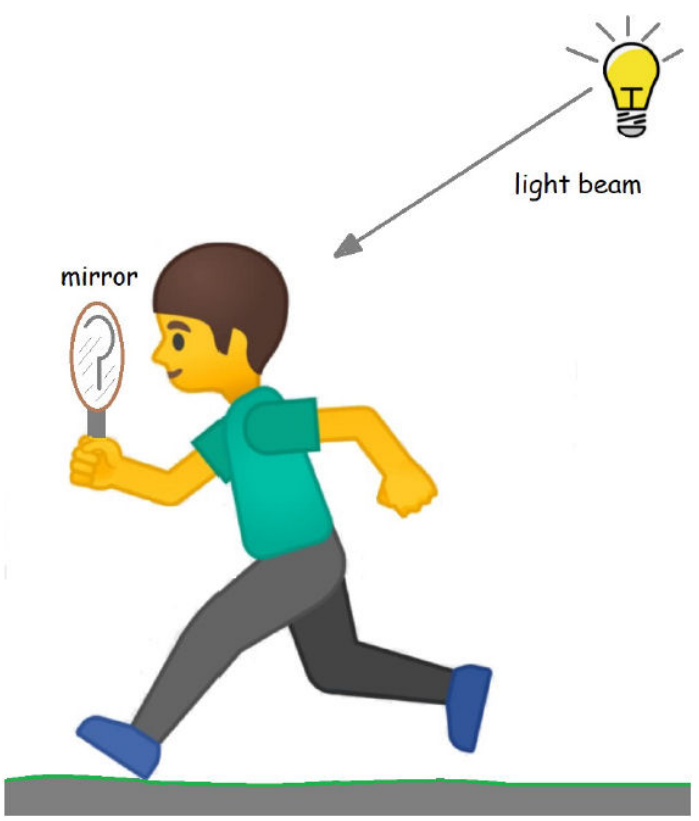

FIGURE 3. Strange mirror.

Step 1 consists, according to the TDS, in a didactic situation, where the teacher assumes the role of mediator and allows the student to experience GTs in didactic situations. The teacher intends to make the student infer the impossibility of using GTs in electrodynamics.

Step 2 consists of the situation of institutionalization of knowledge. It is up to the teacher to show that LTs remove the inconsistencies in electrodynamics. Thus, the teacher shows that GTs are a subset of LTs, formalizing the object of study in a more general framework.

\subsection{Step 2}

In this step 2, the aditic situation begins, which, in light of the TDS, consist of: i) Action Situation: the student must learn a method of solving the problems proposed in step 1 and ii) Formulation Situation: in which the student appropriates knowledge of mathematics applied in physics and establishes a debate through these tools. Also in this step: i) discussing the importance of covariance in topics of classical physics and ii) the transformations between fields.

Brousseau's TDS suggests learning through dialectics. This text contemplates dialectics by making the student understand the validity of GTs and its limitations, leading to the replacement of GTs by LTs.

At first, students should take individual readings of the texts suggested by the teacher. Next, the information must be socialized with one or more colleagues through the exchange of written or oral messages. These steps consist in the dialectic of formulation. This procedure should take place in steps 2 and 3.

The teacher should ask students to talk about the knowledge acquired at the end of steps 2 and 3 . This can be done through a student representing each group. The information must be reinforced or corrected by the teacher. This exchange is part of the didactic contract suggested by Brousseau.

In this step, we will show that Newton's second law does not depend on the inertial frame if supported by the GT. Consequently, the GT also support the laws of dynamics. But the same is not true of Lorentz's strength. The search for a set of transformations that make Lorentz's force not depend on the inertial frame has an impact on Maxwell's equations and the wave equation.

The laws of dynamics are covariant in inertial frames. According to Galilean transformations for inertial frames, it is impossible to detect the uniform movement of one frame about another by any effect on the laws of dynamics [16]. We can verify this statement through the invariance of quantities such as speed, acceleration, and covariance of force. This verification is made if these quantities maintain the same shape when they are written in two different inertial frames $O$ and $O^{\prime}$. Let us observe this invariance in the dynamics through the application of Galileo's transformations in speed, acceleration, and force.

Using vector notation $\vec{x}=(x, y, z)$ and $\vec{v}=(v, 0,0)$ and Galilean transformations for inertial frames:

$$
x^{\prime}=x \pm v t, \quad y^{\prime}=y, \quad z^{\prime}=z, \quad t^{\prime}=t,
$$

we obtain

$$
\begin{aligned}
& v_{x}^{\prime}=v_{x} \pm v, \quad v_{y}^{\prime}=v_{y}, \quad v_{z}^{\prime}=v_{z} \\
& a_{x}^{\prime}=\frac{d^{2} x^{\prime}}{d t^{\prime 2}}=\frac{d^{2} x}{d t^{2}}=a_{x}, \quad a_{y}^{\prime}=a_{y} \quad a_{z}^{\prime}=a_{z},
\end{aligned}
$$

that is, the acceleration has the same values in all references.

Galileo's principle of relativity states that: the laws of mechanics must be the same (i.e., they must remain covariant) in all inertial frames.

To exemplify Galileo's Principle, which states that given two inertial reference frames where Newton's laws are valid, both will be linked by GTs and will be equally good. If $m^{\prime}=m$ :

$$
m \frac{d^{2} x}{d t^{\prime 2}}=m \frac{d^{2} x}{d t^{2}} \rightarrow \vec{F}^{\prime}=\vec{F} .
$$

So two inertial observers measure the same force on a given body. But what about the laws of electrodynamics?

The laws of electrodynamics are expressed through Maxwell's equations, an important law for electrodynamics, is the Faraday-Henry law, expressed in Maxwell's third equation. According to the Faraday-Henry law: $\partial \vec{B} / d t$ creates $\vec{E}$ [17]. In addition to Maxwell's equations, another equation of great importance for electrodynamics, is the equation that defines Lorentz's force:

$$
\vec{F}=q \vec{E}+q(\vec{v} \times \vec{B}) .
$$

The Eq. (4) is, in general, the expression of force in electrodynamics. The question of great importance is: can Lorentz's force be covariant in inertial reference frames? 
The answer to the question is yes, but is not as trivial as in the case of mechanics, that is: The answer is yes, but it is not trivial, as in the case of mechanics. The Lorentz force $(\vec{F})$ consists of the linear combination of the force due to the electric field $\left(\vec{F}_{\|}\right)$and the force due to the magnetic field $\left(\vec{F}_{\perp}\right)$. Hence the Lorentz force covariance ( $\vec{F}$ if, and only if $\vec{F}^{\prime}$ ) must occur from the sum of the components due to the fields.

It is the role of theoretical physics to join efforts to investigate a set of transformations that encompass Galileo's transformations and maintain the covariance of the force vector. This effort became known as the Lorentz covariance or "Principle of Special Relativity" which refers to the property of certain physical equations not changing their forms in inertial frames. It is equivalent to the idea that the laws of physics must take the same form in all inertial frames of reference. This expression for the electrodynamic force occurs in:

$$
\begin{aligned}
& \vec{F}=\frac{d}{d t}\left(\frac{m \vec{v}}{\sqrt{1-\left[\frac{v}{c}\right]^{2}}}\right)=q \vec{E}+q(\vec{v} \times \vec{B}), \\
& \vec{F}^{\prime}=\frac{d}{d t^{\prime}}\left(\frac{m \vec{v}^{\prime}}{\sqrt{1-\left[\frac{v^{\prime}}{c}\right]^{2}}}\right)=q \vec{E}^{\prime}+q\left(\vec{v}^{\prime} \times \vec{B}^{\prime}\right),
\end{aligned}
$$

the term

$$
\gamma=\frac{1}{\sqrt{1-\left(\frac{v}{c}\right)^{2}}}
$$

appears in the definition of physical quantity (see the deduction of Eq. (5) in the Appendix A). Equation (5) makes evident the Lorentz force covariance: the same forms in inertial frames. But note that in addition to the force, there are quantities such as speed and electric and magnetic fields that are involved. Therefore, in electrodynamics, we have to discuss the covariance of the force and the electric and magnetic fields. The speed of electromagnetic radiation is very high compared to the speeds of cars and planes, for example. The invariance for high speeds has been largely explained in the TSR, the high-speed mirror case discusses this high-speed invariance. Then the Lorentz transformations for the force in the inertial frames $O$ and $O^{\prime}$ in which the charge $q$ moves along the $x$ axis are given by

$$
F_{x}=F_{x}^{\prime}, \quad F_{y}=\frac{F_{y}^{\prime}}{\gamma}, \quad F_{z}=\frac{F_{z}^{\prime}}{\gamma} .
$$

To see how the components of the two fields transform, consider an electric and magnetic field and two different inertial frames of reference. The reference frames $O$ and $O^{\prime}$ have coincident axes and relative motion along $x$. Combining them one at a time with the electromagnetic force corresponding to each frame that moves parallel to the speed $\left(O^{\prime}\right)$, we have: $\vec{F}_{\|}^{\prime}=q \vec{E}^{\prime}$, note that in this frame $\vec{B}=0$, because $\vec{v}_{r}=0$. However we have that in the frame $O$, $\vec{F}=q(\vec{E}+\vec{v} \times \vec{B})$, because $\vec{v}_{r} \neq 0$. The Lorentz transformations for the components of the electric field are given by:

$$
\begin{aligned}
& E_{x}^{\prime}=E_{x}^{\prime}, \quad E_{y}^{\prime}=\gamma\left(E_{y}-v B_{z}\right), \\
& E_{z}^{\prime}=\gamma\left(E_{z}+v B_{y}\right) .
\end{aligned}
$$

We can take the direction of relative speed arbitrarily and add the components of the electric field that are parallel $(\|)$ and perpendicular $(\perp)$ to the direction velocity,

$$
\vec{E}_{\|}^{\prime}=\vec{E}_{\|}, \quad \vec{E}_{\perp}^{\prime}=\gamma\left(\vec{E}_{\perp}+[\vec{v} \times \vec{B}]\right) .
$$

But now, in the frame $O^{\prime}$ the charge particle $q$ moves along the $y^{\prime}$ axis being the electromagnetic force $\vec{F}^{\prime}=$ $q \vec{E}^{\prime}+q\left(\vec{v}^{\prime} \times \vec{B}^{\prime}\right)$ and its components:

$$
\begin{aligned}
& F_{x}^{\prime}=q\left(E_{x}^{\prime}+v_{z}^{\prime} B_{z}^{\prime}\right), \quad F_{y}^{\prime}=q E_{y}^{\prime} \\
& F_{z}^{\prime}=q\left(E_{x}^{\prime}+v_{y}^{\prime} B_{x}^{\prime}\right) .
\end{aligned}
$$

In the frame $O$ the magnetic force is also due to $\vec{F}$, taking into account that the speed has components in both the $x$ and $y$ directions and the transformation of speeds between frames is given by

$$
v_{x}=v, \quad v_{y}=\frac{v_{y}^{\prime}}{\gamma}, \quad v_{z}=0 .
$$

With this information, the components of the force become

$$
\begin{aligned}
& F_{x}=q\left(E_{x}+v_{y} B_{z}\right), \quad F_{y}=q\left(E_{y}-v_{x} B_{z}\right), \\
& F_{z}=q\left(E_{z}+v_{y} B_{y}-v_{y} B_{x}\right)
\end{aligned}
$$

With the components of the force in each reference frame and combining once again with the equations of transformations of the force in Eqs. (6) we finally find how the magnetic field is transformed:

$$
\begin{aligned}
& B_{x}^{\prime}=B_{x}^{\prime} \quad B_{y}^{\prime}=\gamma\left(B_{y}+\frac{v}{c^{2}} E_{z}\right) \\
& B_{z}^{\prime}=\gamma\left(B_{z}-\frac{v}{c^{2}} E_{y}\right) .
\end{aligned}
$$

We can also take, as before, the direction of the relative speed in an arbitrary way and add the components of the electric field that are parallel $(\|)$ and perpendicular $(\perp)$ to the direction of the speed leaving the more general expression:

$$
\vec{B}_{\|}^{\prime}=\vec{B}_{\|}, \quad \vec{B}_{\perp}^{\prime}=\gamma\left(\vec{B}_{\perp}-[\vec{v} \times \vec{E}]\right) .
$$

The results for the transformation of the electric $\vec{E}$ and magnetic $\vec{B}$ fields in Eqs. (8) and (10) contain important information. It is possible to see that the components that are parallel to the relative speed of the reference frame do not change when changing the frame of reference, however, the perpendicular components suffer a mixture of electric and magnetic fields. These transformations, in the context of the Theory of Relativity, introduce the concept of relativity in the 
electric and magnetic fields, showing their dependence on the relative movement of the observer.

In this way, with the connection established through the Lorentz transformations of the fields, it makes no sense to think of them separately, since together they form what we call the electromagnetic field. Thus, a field that is seen to be only electric $\vec{E}$ or magnetic $\vec{B}$ in one frame, is observed in another frame as having components $\vec{E}$ and $\vec{B}$. Covariance cannot be understood with the electric and magnetic fields separately, the covariance occurs about the electromagnetic field. Note that the Lorentz force covariance led to the electromagnetic field covariance.

The transformations between fields solves the problem of covariance against inertial references in Maxwell's equations, for example, in the third equation (Faraday- Henry's law),

$$
\vec{\nabla} \times \vec{E}=-\frac{\partial \vec{B}}{\partial t} .
$$

We have that:

- In the frame $O$ :

$$
\frac{\partial}{\partial x} E_{z}-\frac{\partial}{\partial z} E_{x}=\frac{\partial}{\partial t} B_{y} .
$$

- In the frame $O^{\prime}$ :

$$
\frac{\partial}{\partial x^{\prime}} E_{z}^{\prime}-\frac{\partial}{\partial z^{\prime}} E_{x}^{\prime}=\frac{\partial}{\partial t^{\prime}} B_{y}^{\prime} .
$$

Equations (7) and (9) take the third equation (Faraday- Henry law), in the frame $O^{\prime}$ :

$$
\begin{gathered}
\frac{\partial}{\partial x^{\prime}} E_{z}^{\prime}-\frac{\partial}{\partial z^{\prime}} E_{x}^{\prime}=\frac{\partial}{\partial t^{\prime}} B_{y}^{\prime} \\
\frac{\partial}{\partial x^{\prime}}\left(\gamma\left[E_{z}+v B_{y}\right]\right)-\frac{\partial}{\partial z^{\prime}} E_{x} \\
=\frac{\partial}{\partial t^{\prime}}\left(\gamma\left[B_{y}+\frac{v}{c^{2}} E_{z}\right]\right) .
\end{gathered}
$$

The detailed deductions for the covariance of Maxwell's equations against Lorentz's transformations can be seen in Ref. [18].

Shockingly, Maxwell showed that the equations governing electrodynamics could also be combined in a way that would provide us with the wave equation for the electric and magnetic field vectors [19]. A consequence of the wave equation is that it is not invariant in the face of transformations (Eqs. (2)). However, it is invariant for

$$
\begin{aligned}
x^{\prime} & =\gamma(x \pm v t), \quad y^{\prime}=y, \quad z^{\prime}=z \\
t^{\prime} & =\gamma\left(t-\frac{v x}{c^{2}}\right) .
\end{aligned}
$$

These transformations are known as Lorentz transformations.

\subsection{Step 3}

Step 3 consists of removing the inconsistencies presented in step 1 through the Lorentz transformations. In TDS, steps 3 and 4 are where the Validation Situation occurs through the removal of inconsistencies (step 3) and the technologies extracted from the new knowledge (step 4).

The intrinsic relationship between the electric and magnetic fields, which is directly related to the covariance of the Lorentz force, is the key to answer the possible inconsistency raised in situations 1 and 2. Two observers in two inertial frames can't see two distinct movements for the charge. There is a single cause for emf in situation 2 .

The covariance of the wave equation leads to the invariance of the speed of electromagnetic radiation (light), so the value of the speed of light does not depend on the inertial frame. This is the key to responding to possible inconsistencies in situations 3 and 4.

The Michelson and Morley experiment supports the invariance of the speed of light. Therefore, there is no support for the ether hypothesis. If the speed of light is independent of the inertial frame, then the boy will see his face reflected even at high speed.

\subsubsection{Magic carpet}

According to Professor Cláudio José de Holanda Cavalcante, IF-UFRGS [20]:

The basic error that leads to the false conclusion that the boy would not see the sphere accelerate is to disregard the transformation of the electric and magnetic fields between different inertial references.

Assuming that the girl's frame with $O$ and the boy's frame as $O^{\prime}$, we have for the fields:

$$
\begin{aligned}
\vec{E}^{\prime} & =\vec{v} \times \vec{B}, \\
\vec{B}^{\prime} & =\vec{B} .
\end{aligned}
$$

Equation (13) states that the magnetic fields experienced by the girl $(O)$ and the boy $\left(O^{\prime}\right)$ are the same. Equation (12) states that the electric field experienced by the boy $\left(O^{\prime}\right)$ is perpendicular to the magnetic field, so that $\vec{E}^{\prime}$ exerts a force on the sphere.

The results of Eqs. (12) and (13) can be obtained through the Lorentz transformations, more explicitly:

$$
\begin{aligned}
\vec{E}^{\prime} & =\gamma(\vec{v} \times \vec{B}), \\
\vec{B}^{\prime} & =\gamma \vec{B}
\end{aligned}
$$

with

$$
\gamma=\frac{1}{\sqrt{1-\left(\frac{v}{c}\right)^{2}}}
$$

and for $v \ll c$, we have $\gamma \approx 1$, [21]. 
Knowing that: i) $\vec{E}=\vec{E}_{\|}+\vec{E}_{\perp}$ and $\vec{B}=\vec{B}_{\|}+\vec{B}_{\perp}$ with a velocity component parallel to $\vec{E}_{\|}$we have $\cos (\theta)=\left|\vec{E}_{\|}\right| / E$ between $\vec{E}$ and $\vec{E}_{\|}$or $\vec{v}$, from these we have: ii) $\vec{E}_{\|}=$ $E \cos (\theta)(\vec{v} / v)$. In a similar way: iii) $\vec{B}_{\|}=(\vec{B} \cdot \vec{v})(\vec{v} / v)$. Combining the last results with the relativistic transformations between fields $(8)$ and (10), we arrive at:

$$
\begin{aligned}
\vec{E}^{\prime} & =\gamma(\vec{E}+\vec{v} \times \vec{B})-(\gamma-1) \frac{\vec{E} \cdot \vec{v}}{v^{2}} \vec{v}, \\
\vec{B}^{\prime} & =\gamma\left(\vec{B}-\frac{\vec{v} \times \vec{E}}{c^{2}}\right)-(\gamma-1) \frac{\vec{B} \cdot \vec{v}}{v^{2}} \vec{v},
\end{aligned}
$$

As $\vec{E}=0$ because the sphere is in the reference frames $O^{\prime}$ (in the boy's hands), and since $v \ll c$, then $\gamma$ tends to 1 and $\vec{E}^{\prime}=\vec{v} \times \vec{B}$. Putting $\vec{E}=0$ in the relation of $\vec{B}^{\prime}$ and $\vec{B} \cdot \vec{v}=0$, since $\vec{B}$ is perpendicular to $\vec{v}$, so $\vec{B}^{\prime}=\vec{B}$ with $\gamma$ tending to a $1[20]$.

According to Alberto Gaspar [9]:

The electromagnet generates not only a magnetic field, but an electromagnetic field, whose vectors magnetic and electric fields are interrelated according to the relativistic transformations.

Both the girl and the boy will see the same movement for the sphere, as in both references the sphere will be affected by the electric and magnetic field. The sphere will not be at rest in relation to the boy.

\subsubsection{Spiral on rails}

As in the case of the magic carpet, we will suppose that the observer who sees the movement is the $O$ frame and the observer who is in motion is the $O^{\prime}$ frame.

From the Lorentz transformations for the electric and magnetic field we can obtain a better understanding of what occurs in the $O^{\prime}$ frame. For that, it is convenient that we use the transformations of the fields proposed in Eqs. (8) and (10). Recall that in the frame $O$, the electromagnetic field manifests as purely magnetic, $\vec{B} \neq 0$ and $\vec{E}=0$.

From the transformations we obtain:

$$
\vec{E}_{\|}^{\prime}=0, \quad \vec{E}_{\perp}^{\prime}=\gamma(\vec{v} \times \vec{B}),
$$

and

$$
\vec{B}_{\|}^{\prime}=\vec{B}_{\|}, \quad \vec{B}_{\perp}^{\prime}=\gamma \vec{B}_{\perp}
$$

with

$$
\vec{v} \times \vec{B}=\vec{v} \times \vec{B}_{\perp}=\frac{\vec{v} \times \vec{B}_{\perp}^{\prime}}{\gamma}=\frac{\vec{v} \times \vec{B}^{\prime}}{\gamma},
$$

we find that:

$$
\vec{E}=\vec{v} \times \vec{B} .
$$

The result found shows that in fact what the electron experiences in the frame $O^{\prime}$ is an electric field, where $\vec{v}$ is the relative speed between the frames. And, it is this electric field that will give rise to an emf. Another important fact is that the force acting on the electric charge is given by the same Lorentz force equation used in the frame $O, \vec{F}=q(\vec{v} \times \vec{B})$. It is important to note that in the $O^{\prime}$ frame there is both an electric and magnetic field, in contrast to what the observer in the $O$ frame considers to be only a magnetic field.

Observer $O$ perceives the movement of the charge $q$ on the coil, and consequently a Lorentz force. The electromotive force will be given by:

$$
\begin{aligned}
\varepsilon & =\frac{1}{q} \int \vec{F} \cdot d \vec{l}=\int \vec{E} \cdot d \vec{l} \\
& =\int(\vec{v} \times \vec{B}) \cdot d \vec{l}=-v B l .
\end{aligned}
$$

Note that:

- $\vec{v}$ perpendicular to $\vec{B}$;

- The square loop has side $l$;

- $\vec{E}$ is obtained from Eq. (16);

- The negative sign is due to $\vec{v}$ being in the negative direction.

The observer $O^{\prime}$ does not perceive the movement of the charge $q$ on the coil, and consequently, there is no Lorentz force. But from the magnetic field flow

$$
\Phi(B)=\int \vec{B} \cdot d \vec{A}=B l s,
$$

being $s$ only the part of the loop that enters the field.

According to the Faraday-Henry law $\partial \vec{B} / \partial t$ creates $\vec{E}$, then the emf:

$$
\epsilon=\int \vec{E} \cdot d \vec{l}=-\frac{d \Phi(B)}{d t}=-B l \frac{d s}{d t}=B l v .
$$

With this situation, it becomes clear by using relativity that it does not make sense to think of the independent existence of electric and magnetic fields, and that these assume a relative character. Thus, both an observer in $O$ and $O^{\prime}$ are correct in their respective observations, with no preferable reference frame with respect to any other. What one observer interprets to be a magnetic process, another observer interprets to be an electrical process.

\subsubsection{Ether}

Ether emerged as a reference frame needed for the laws of electrodynamics and, in the at the end of the 19th century, Michelson and Morley (1887) devised an experiment to measure the speed of the Earth with respect to ether [22]. Figure 4 illustrates the Michelson and Morley interferometer.

According to Baldiotti [24]: 


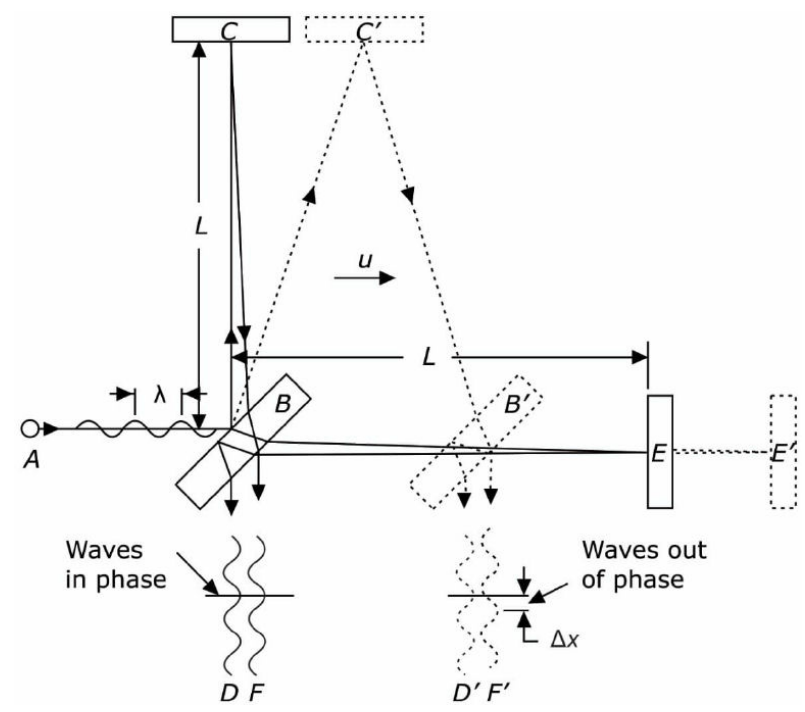

FIGURE 4. Michelson and Morley interferometer [23].

- In this apparatus, a coherent beam of light is divided into two beams by a partially silver plate. The two beams are reflected by mirrors that are at the same distance $L$ and recombine. If we imagine that the light propagates in the ether and that it is at rest concerning the apparatus, both beams will travel the same distance and recombine constructively. Now, if the light propagates in the ether (as the sound propagates in the air) it is the ether that moves with a speed $v$ concerning the apparatus, the beams will travel different distances and recombine out of phase.

The distance between plate $B$ and the plate $C$ is given by

$$
d_{c}^{2}=L^{2}+\left(v t_{c}\right)^{2},
$$

where $v$ is the speed of propagation of ether and $t_{c}$ is the time for the electromagnetic radiation travel the $A B$ distance. Since the speed of radiation $c$, we have to: $d_{c}=c t_{c}$. Combining the two previous equations, we have

$$
t_{c}=\frac{L / c}{\sqrt{1-\left(\frac{v}{c}\right)^{2}}}=\gamma \frac{L}{c},
$$

where

$$
\gamma=\frac{1}{\sqrt{1-\left(\frac{v}{c}\right)^{2}}}
$$

is the Lorentz factor. The distance traveled by the radiation perpendicularly to $v$ is given by:

$$
L_{\perp}=2 d_{c}=2 \gamma L .
$$

The distance between plate $B$ and the plate $E$ is given by

$$
d_{B E}=L+v t_{B E} \quad \therefore \quad d_{B E}=L\left(1+\frac{v}{c-v}\right),
$$

and the distance for the beam to return:

$$
d_{E B}=L-v t_{E B} \quad \therefore \quad d_{E B}=L\left(1-\frac{v}{c+v}\right) .
$$

The total distance is given by:

$$
\begin{aligned}
L_{\|} & =d_{B E}+d_{E B} \\
& =L\left(2+\frac{v}{c}\left[\frac{1}{1-\frac{v}{c}}-\frac{1}{1+\frac{v}{c}}\right]\right)=2 L \gamma^{2} .
\end{aligned}
$$

The different values of $L_{\perp}$ and $L_{\|}$make it possible to detect variations in the ether speed through the interference fringes in the composition of the outgoing beams [24]. The experiment did not detect any difference in the speed of the beams; hence, the conclusion was that there is no relative speed between Earth and ether. Given the evidence from the experience of Michelson and Morley, we see that a model of a reference frame associated with ether for electrodynamics is unsustainable.

\subsubsection{High speed mirror}

At this point, we already know that Galileo's transformations lead to inconsistencies in electromagnetism, and the equation that describes the propagation of the electromagnetic wave is not covariant in the face of Galileo's transformations (Eq. (2)), but it is covariant in the face of Lorentz's transformations (Eq. (11)). The deduction of Lorentz transformations starts from the hypothesis that the speed of electromagnetic radiation is invariant in inertial frames.

Let $O$ be the frame at rest, and let $O^{\prime}$ be the frame in a straight, uniform motion. Consider the case where the origins coincide at $t=t^{\prime}=0$ and $O^{\prime}$ is in motion with velocity $v$ parallel to the $x$ axis. If the particle has velocity $v_{x}$ in frame $O$, its velocity in frame $O^{\prime}$ is

$$
v_{x}^{\prime}=\frac{d x^{\prime}}{d t^{\prime}}=\frac{d x}{d t}-v=v_{x}-v,
$$

assuming $x^{\prime}=k(-v t)$ and $t^{\prime}=a(t-b x)$.

After a time $t$, the light pulse reaches point $\mathrm{A}$ at $r=c t$, of the frame $O$, so that:

$$
\begin{gathered}
x^{2}+y^{2}+z^{2}=c^{2} t^{2} \\
x^{\prime 2}+y^{\prime 2}+z^{\prime 2}=c^{2} t^{\prime 2}
\end{gathered}
$$

After some algebraic manipulation, we recover Eqs. (11) from which the formulas for velocity-addition are derived. Suppose that a particle travels a distance $d x$ in an interval $d t$, in the frame $O$. The velocity in the frame $O^{\prime}$ is obtained from

$$
v^{\prime}=\frac{V-v}{1-\frac{v}{c^{2}} V},
$$

where, $V$ is the speed of a massive structure in relation to $O$ and $V^{\prime}$ be the speed of a massive structure in relation to $O^{\prime}$ 
(see Appendix A). Considering an electromagnetic wave that propagates in the direction of the $x$ axis $(V=c)$, then:

$$
v^{\prime}=\frac{c-v}{1-\frac{v}{c}}=c .
$$

From the law of speed addition we can find in a simple way the same result of the Fresnel's theory. Calling the speed of a massive structure in relation to $O$, with refractive index $n$, of $V=c / n$, if the speed of the medium is opposite to the speed of light. From the speed addition we have:

$$
\frac{\frac{c}{n}+v}{1-\frac{v c}{n c^{2}}} \approx \frac{c}{n}+\left(1-\frac{1}{n^{2}}\right) v,
$$

for $v^{2} \ll n c$, where $1 / n^{2}=\rho_{e} / \rho_{f}$.

The previous equation confirms the results predicted in the Fresnel and Stokes models without the need for the existence of ether.

The speed of electromagnetic radiation is invariant for all inertial frames, so it is impossible for the boy not to see himself in the mirror.

\subsection{Step 4}

In TDS, the adidactic situation is ended in the validation stage. One way to validate the dynamics of action and validation is to understand the technologies and applications derived from the solutions of the inconsistencies. Here, we have two derived technologies: GPS and physics. Step 4 in the TDS deals with the situation of validation and institutionalization.

GPS and nuclear physics are part of students' daily lives, so these technologies give meaning to all efforts to understand the importance of LTs in electrodynamics.

In the last situation, the teacher must clarify the objectives through the knowledge acquired in the previous steps. Institutionalization, in the teaching of mathematics according to Guy Brousseau, is accomplished through the formalization and generalization of knowledge.

The present work has as an objective to implement the TDS in theoretical physics and for that we have to differentiate the knowledge acquired in Mathematics from the knowledge acquired in Physics. In Physics, the guiding principle of the theory plays an important role, for example, we have the principles of conservation. The idea of the postulate of a physical theory arises to guide and direct the whole theory.

The situation of institutionalization of work related to Physics calls attention to add principles and postulates to the mathematical formulation, thus in the generalization of the knowledge of topics in theoretical physics, they are also associated with principles and postulates.

The discussion of problematizations is closely related to the covariance of the laws of physics in inertial references. Therefore, both the observer at rest and the observer in uniform rectilinear motion must view the phenomena of electromagnetism in the same way. This intuition leads to the understanding of STR which is based on two postulates:
1. The laws of physics (mechanics and electrodynamics) take the same form for any inertial reference;

2. The speed of light in a vacuum is $c$ for any inertial frame regardless of the movement assumed by the source.

The first postulate says that it is not possible, through purely mechanical or electromagnetic experiments, to infer to the observer what the state of movement of the reference system is. As for the second postulate, it is an immediate consequence of the first, otherwise, there would be the possibility of distinguishing an inertial referential from another also inertial, which would imply the existence of an absolute system.

Both postulates are supported by LTs, which relate distances and times. This leads us to important results that were very well understood by Einstein. The first is the phenomenon of time dilation, observed when two inertial observers in relative motion decide to measure the time interval between two events. Therefore, we have to,

$$
\Delta t=\frac{\Delta t^{\prime}}{\sqrt{1-\left(\frac{v}{c}\right)^{2}}} .
$$

Another important result, closely linked to time dilation is the contraction of the dimensions of objects in the direction of analysis of the movement known as contraction of lengths:

$$
l=\left(\sqrt{1-\frac{v^{2}}{c^{2}}}\right) .
$$

In Eq. (22), $\Delta t^{\prime}$ represents the time interval between two events measured by an observer in frame $O^{\prime}$, and $\Delta t$ the time interval measured by an observer in frame $O$ that sees frame $O^{\prime}$ moving in uniform motion with velocity $v$. In Eq. (23), the length of the bar measured in the frame in which it is at rest is called its length called $l_{0}$.

In addition to temporal expansion and contraction of spaces, the conceptual change also occurs with mass, moment, and energy within the STR.

We can highlight two applications for the theory of relativity: GPS and nuclear energy.

\subsection{Global Positioning System}

The Global Positioning System (GPS) is a localization tool widely found today. It is used for navigation of vessels, airplanes, and vehicles and is at hand with computers and smartphones.

This technology became possible due to the efforts of aerospace engineering and telecommunications, basically a system of satellites that emit radio signals synchronized by atomic clocks corrected due to time dilation. We have here three areas of physics that communicate: electrodynamics (radio signals), mechanics quantum (atomic clocks), and STR 


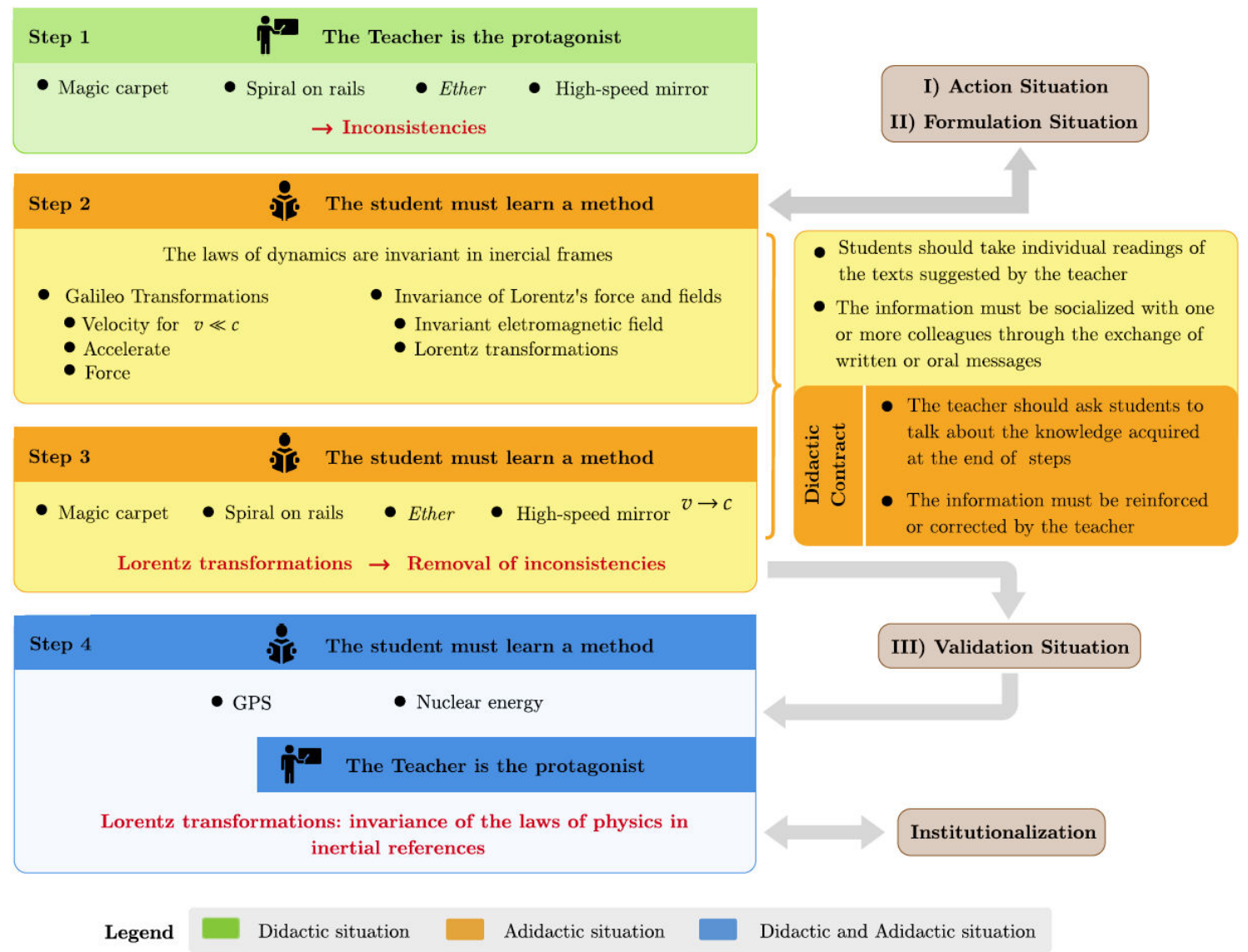

FIGURE 5. Summary of the didactic proposal worked on the proposed topics of theoretical physics.

(time dilation). This communication requires a understanding of the mathematical equations that make possible the accuracy of information via GPS [25].

A given information from a single GPS receiver requires four pieces of information via four satellites modeled using a system of four equations:

$$
\begin{aligned}
& \sum_{i=1}^{4}\left(\left[x-x_{i}\right]^{2}+\left[y-y_{i}\right]^{2}+\left[z-z_{i}\right]^{2}\right) \\
& =\sum_{i=1}^{4} c^{2}\left(t-t_{i}\right)^{2} .
\end{aligned}
$$

We know that the speed of the radio signal is $c=300,000$ $\mathrm{km} / \mathrm{s}$ so that an accuracy of $5 \mathrm{~m}$ in position requires an accuracy of 5 nanoseconds [25]. The term $\left(t-t_{i}\right)^{2}$ should be corrected due to the time dilation for physical entities that move at high speeds.

\subsection{Nuclear energy}

A direct consequence of STR is the equivalence between mass and energy. This made possible the use of a new form of energy, namely: nuclear or atomic energy. Nuclear energy accounts for $16 \%$ of the energy produced in the world today [26]. Nuclear plants are more concentrated in the north- ern hemisphere, mainly in western Europe and North America. The electricity generated in a nuclear plant comes from the heat produced in the nuclear fission process. In this, the atomic mass is divided releasing energy.

The relationship between mass and energy is expressed by the equation:

$$
E=\frac{m_{0}^{2}}{\sqrt{1-\left(\frac{v}{c}\right)^{2}}}-m_{0} c^{2} .
$$

Since the speed of light $c$ is invariant, we have that energy is a function of the resting mass: $E=f\left(m_{0}\right)$. In such a way, a particle, although at rest, has the energy that is associated with mass. "So we have to replace the classic conservation laws, separately from mass and energy, with a single law of conservation of total relativistic energy: the total relativistic energy of an isolated system remains constant" [27].

The dynamics proposed to be worked on in the TSD are summarized in Fig. 5. Basically, the dynamics consist of didactic and adidactic situations in which the milieu deals with topics in theoretical physics that require an advanced knowledge of mathematics. Figure 5 shows that Galileo's transformations support covariance within dynamics, but that they do not support covariance in electromagnetism, this is contextualized utilizing inconsistencies. The effort to make Lorentz's 
force invariant led to the covariance of the electromagnetic field and Lorentz's transformations. From a new set of transformations, inconsistencies are removed and new technologies are created. The physics that treats objects at very high speeds is modern physics and must be treated through the Lorentz transformations.

A good exercise is to approximate the speed much less than that of light in Lorentz transformations. The student must be encouraged to understand that Galileo's set of transformations is embedded in Lorentz's set of transformations, therefore modern physics encompasses classical physics. Therefore, there is no substitution, but a complement.

\section{Conclusion}

A good exercise is to make the speed much less than that of light in Lorentz transformations. The student must be provoked to understand that Galileo's set of transformations is inserted in Lorentz's set of transformations, therefore modern physics encompasses classical physics. Therefore, there is no substitution, but a compliment.

For this we started from four situations that lead to the following questions: i) can different observers observe different movements for the charge? ii) can the same electromotive force be caused by different fields? iii) can the same value for the speed of light, in different inertial reference frames, make the ether hypothesis unsupported? v) how can a boy look in the mirror and not see his face?

The answers to these questions cannot be found with the use of the set of transformations commonly used in dynamics (Galileo transformations). The answer depends on the understanding of covariance in electrodynamics, thus resulting in a new set of transformations (Lorentz transformations) that answer the initial questions and are key understanding of everyday technologies (GPS and nuclear physics).

This paper suggests a set of practices centered on TDS and a text for use in university courses for the training of physics and mathematics teachers. In the set of practices, we distinguish didactic and adidactic moments guided by a narrative from problem situations solved using previous and recently acquired knowledge of physics.

The difficulty of understanding electrodynamics and the special theory of relativity, as well as the relationships between these two theories, calls for the application of pedagogical methods. We understand that TDS can be applied to theoretical physics, for this we suggest a narrative and a set of practices.

\section{Appendix A}

The transformation of forces into inertial references, according to STR, depends on the transformation of velocities and changes in the definition of momentum. Let the inertial frame $O:(x, y, z, t)$ and the inertial frame $O^{\prime}:\left(x^{\prime}, y^{\prime}, z^{\prime}, t^{\prime}\right)$ with constant speed $v$ in the $x$ direction, let $V$ be the speed of a structure massive in relation to $O$ e let $V^{\prime}$ be the speed of a massive structure in relation to $O^{\prime}$. According to the transformations:

$$
d x^{\prime}=\frac{d x-v d t}{\sqrt{1-\left(\frac{v}{c}\right)^{2}}},
$$

and

$$
d t^{\prime}=\frac{d t-\frac{v}{c^{2}} d x}{\sqrt{1-\left(\frac{v}{c}\right)^{2}}},
$$

Therefore, the transformation of velocities, according to $\mathrm{STR}$, is given by

$$
v^{\prime}=\frac{d x^{\prime}}{d t^{\prime}}=\frac{V-v}{1-\frac{v}{c^{2}} V^{\prime}},
$$

for $V=d x / d t$.

The moment must be redefined so that the energy conservation principle remains valid for the STR. This is possible by admitting that the mass may vary in inertial references. The mass is then defined by $m=m_{0} / \sqrt{1-(v / c)^{2}}$, where $m_{0}$ is the resting mass. The force is then defined, according to the new moment by

$$
\begin{aligned}
F & =\frac{d}{d t}\left(\frac{m_{0} V}{\sqrt{1-\left(\frac{v}{c}\right)^{2}}}\right) \\
& =\frac{m_{0} \frac{d V}{d t}}{\sqrt[3 / 2]{1-\left(\frac{v}{c}\right)^{2}}},
\end{aligned}
$$

in reference $O$. Similarly, we have for reference $O^{\prime}$ :

$$
F^{\prime}=\frac{m_{0} \frac{d V^{\prime}}{d t}}{\sqrt[3 / 2]{1-\left(\frac{v^{\prime}}{c}\right)^{2}}},
$$

and knowing that $d / d t^{\prime}=\left(d t / d t^{\prime}\right)(d / d t)$ and that $d t / d t^{\prime}=$ $\sqrt{1-(v / c)^{2}} /\left(1-\left[v / c^{2}\right] V\right)$, we have:

$$
F^{\prime}=\frac{m_{0} \frac{d V^{\prime}}{d t}}{\sqrt[3 / 2]{1-\left(\frac{v^{\prime}}{c}\right)^{2}}} .
$$

The relationship between Eqs. (A.4) and (A.5) depends on the relationship between $d V / d t$ and $d V^{\prime} / d t$. Knowing that $d V / d t=a, d V^{\prime} / d t=a^{\prime}$ and (A.3), where $a$ and $a^{\prime}$ are accelerations in relation to $O$ and $O^{\prime}$ respectivamente, respectively, we have that:

$$
a^{\prime}=\frac{a\left(1-\frac{v^{2}}{c^{2}}\right)}{\left(1-\frac{v}{c^{2}} V\right)^{2}} .
$$

The consideration that the massive structure is at rest in relation to $O^{\prime}\left(V^{\prime}=0\right)$, leads to $V=v$ and:

$$
F^{\prime}=\frac{m_{0} a}{\sqrt[3 / 2]{1-\left(\frac{V^{\prime}}{c}\right)^{2}}}=F,
$$

being Eq. (A.7) the invariance of the force in STR. 


\section{Acknowledgments}

The authors would like to thank the Ph.D. Professors José Carlos Freire Machado and Edson Akira Asano, in addition to student Tiago de Oliveira from the extinct Environmental Physics course at UFOPA for his contributions to this paper.

1. J. M. F. Bassalo, Nascimentos da Física (3500 a.C.-1900 a.D.) (Editora da Universidade Federal do Pará, Belém, 1996).

2. J. C. Maxwell, A Treatise on Eletricity and Magnetism (Clarendon Press, Oxford, 1873).

3. H. A. Lorentz, Electromagnetic phenomena in a system moving with any velocity smaller than that of light, Proc. Acad. Sciences Amsterdam 6 (1904) 809.

4. A. Einstein, Über die von der molekularkinetischen Theorie der Wärme geforderte Bewegung von in ruhenden Flüssigkeiten suspendierten Teilchen, Ann. Phys. 322 (1905) 549. https: //doi.org/10.1002/andp.19053220806.

5. H. G. Hertz and M. G. Doncel, Heinrich Hertz's laboratory notes of 1887, Arch. Hist. Exact Sci. 49 (1995) 197, https: //doi.org/10.1007/BF00376092

6. G. Brousseau, Fondements et méthodes de la didactique des mathématiques, Rech. Didact. Math. 7 (1986) 33, https://revue-rdm.com/1986/ fondements-et-methodes-dela/

7. P. J. Magalhã es Teixeira and C. C. Manso Passos, Um pouco da Teoria das es Didáticas (TSD) de Guy Brousseau, Zetetike 21 (2014) 155, https://doi.org/10.20396/ zet.v21i39.8646602

8. R. Resnick, Introdução a Relatividade Especial (Editora Polígono, São Paulo, 1971).

9. A. Gaspar, Física: Eletromagnetismo e Física Moderna (Editora Ática, São Paulo, 2002), v. 3.

10. D. J. Griffiths, Introduction to Electrodynamics (Cambridge University Press, 2017), 4th ed.

11. J. R. Reitz, F. J. Milford e R. W. Christy, Foundations of Electromagnetic Theory (Addison-Wesley, 2008), 4th ed.

12. C. Kittel, W. D. Knight e M. A. Ruderman, Curso de Física de Berkeley: Mecánica (Edgard Blucher, São Paulo, 1973). Vol. 1.

13. R. de A. Martins, Sobre o artigo A dinámica relativística antes de Einstein, Rev. Bras. Ens. Fis. 27 (2005) 313, https:// doi.org/10.1590/S1806-11172005000300002

14. H. M. Nusseszveig, Curso de Física Básica: Ótica, Relatividade e Física Quántica (Edgard Blücher, São Paulo, 1998), 1 ed.
15. R. de A. Martins, O éter e a óptica dos corpos em movimento: a teoria de Fresnel e as tentativas de detecçã o do movimento da terra, antes dos experimentos de Michelson e Morley (1818-1880), Cad. Bras. Ens. Fis. 29 (2012) 52, https: //doi.org/10.5007/2175-7941.2012v29n1p52

16. H. M. Nusseszveig, Curso de Física Básica: Mecânica Básica (Edgard Blücher, S ao Paulo, 2002), 4 ed.

17. M. I. Nobre Ota, Eletromagnetismo e Relatividade: Continuidade formal-ruptura conceitual, Rev. Bras. Hist. Mat. 5 (2005) 27, https://doi.org/10.47976/ RBHM2005v5n1027-42.

18. P. R. Ferreira Fragata, Tópicos da Teoria da Relatividade. Dissertação de Mestrado, Universidade de Açores, (2013).

19. P. A. Tipler e G. Mosca, Física para Cientistas e Engenheiros Eletricidade, Magnetismo e Ótica (LTC, Rio de Janeiro, 2011), 6 ed.

20. Centro de Referência para o Ensino de Física, Eletromagnetismo e relatividade no livro do Gaspar, available in htts://www.if.ufrgs.br/ novocref/?contact-pergunta=eletroma gnetismo-e-relatividade-nolivro-do-gaspar

21. M. Le Bellac and J. M. Lévy-Leblond, Galilean electromagnetism, Nuovo Cimento B 14 (1973) 217, https://doi. org/10.1007/BF02895715

22. A. A. Michelson and E. W. Morley, Am. J. Sci. 34 (1887) 333.

23. R. Feynman, The Feynman Lectures on Physics, (Basic Books, New York, 2010), v. 1.

24. M. C. Baldiotti, Física Moderna I - Parte A: a Teoria da Relatividade, available in htt://www.uel.br/pessoal/ baldiotti/2FIS026A.pdf

25. J. Natário, O GPS e a Teoria da Relatividade, available in htts://www.math.tecnico.ulisboa.pt/ jnatar/lectures/GPS.pdf

26. C. Batista, Energia Nuclear, htts://www.todamateria. com.br/energia-nuclear/

27. S. Vieira, A. Barros, I. Araújo e J. C. T. Oliveira, Uma comparação entre deduçoes da ecuação $E=m c^{2}$, Rev. Bras. Ens. Fis. 26 (2004) 93, https://doi.org/10.1590/ S1806-11172004000200003 